\title{
INTERVENCIÓN COMUNITARIA DESDE LA EDUCACIÓN FÍSICA, UN PROCESO TRANSFORMADOR DE REALIDADES SOCIALES
}

\section{COMMUNITY INTERVENTION FROM PHYSICAL EDUCATION, A TRANSFORMING PROCESS OF SOCIAL REALITIES}

\section{Andrés Fernando Balcázar Vega ${ }^{1}$}

\section{Nicolás Guarnizo Carballo²}

\section{Fernando Campos Polo ${ }^{3}$}

\section{RESUMEN}

El presente artículo condensa los elementos más representativos de un ejercicio de carácter investigativo que permitió demostrar de qué manera los procesos de intervención comunitaria desarrollados a partir del proyecto OCÚPATE de la Universidad de la Llanos (Colombia) han incidido en la transformación social del

1 Andrés Fernando Balcázar Vega Licenciado en Educación Física y Deportes Especialista en Recreación Ecologica, Universidad de los Llanos

$2 \quad$ ORCID: 0000-0002-3682-2722

abalcazar@unillanos.edu.co

Nicolás Guarnizo Carballo Licenciado en Educación Física y Deportes Maestria en Educación Internacional Universidad de los Llanos. ORCID: 0000-0002-2969-4523

Nicolas.guarnizo@unillanos.edu.co

3 Fernando Campos Polo

Licenciado en Educación Física y Deportes Doctor

en Educación Universidad de los Llanos.

ORCID: 0000-0002-1819-5497 fcampos@unillanos.edu.co barrio la Madrid del municipio de Villavicencio. La investigación realizada adoptó un método descriptivo de corte cualitativo en la que mediante la implementación de técnicas de entrevistas y revisión documental se recolecto información suficiente que al ser contrastada y analizada arrojó resultados que avalan la continuidad de las dinámicas de intervención en la comunidad mencionada. Los resultados obtenidos demuestran que la intervención comunitaria desarrollada a la luz de la responsabilidad social de la Universidad se enfoca en tres elementos particulares, la ocupación lúdica y productiva del tiempo libre, el fortalecimiento del capital humano y la potenciación del capital social, no siendo estos los únicos aspectos favorecidos en el proceso, pero si los más relevantes, es en este 
sentido en que las conclusiones dan muestra de la manera en que los procesos de intervención comunitaria adelantados mediante el proyecto Ocúpate de la Universidad de los Llanos vienen contribuyendo en el desarrollo social del barrio la Madrid de Villavicencio.

Palabras clave: capital humano, capital social, tiempo libre, intervención comunitaria.

\section{ABSTRACT}

This article summarizes the most representative elements of an investigative exercise that will demonstrate how the community intervention processes developed from the OCÚPATE project of the Universidad de los Llanos (Colombia) have influenced the social transformation of the Madrid neighborhood. from the municipality of Villavicencio. The research carried out adopted a qualitative descriptive method in which, through the implementation of interview techniques and documentary review, sufficient information is collected that, when contrasted and analyzed, yielded results that support the continuity of the dynamics of community intervention in the mentioned community. The results showed that the community intervention developed in the light of the social responsibility of the University focuses on three particular elements, the recreational and productive occupation of free time, the strengthening of human capital and the empowerment of social capital, not being these the The only aspects favored in the process, but if the most relevant, it is in this sense that the conclusions show how the community intervention processes carried out through the Ocúpate project of the Universidad de los Llanos have been contributing to social development from the Madrid neighborhood of Villavicencio.

Keywords: human capital, social capital, free time, community intervention.

\section{INTRODUCCIÓN}

La intervención comunitaria desarrollada por la institucionalidad de diferentes Universidades en el contexto internacional, se sustenta a partir de la responsabilidad social que afloran desde sus funciones sustantivas, permitiendo un mayor acercamiento entre el ámbito académico vibrante en el seno de cada alma mater y los distintos grupos sociales existentes entre los sectores constituyentes del tejido social. En este sentido las universidades especialmente públicas, han jugado un papel muy importante que no solo se evidencia desde la óptica de la docencia y la investigación, -que sin ser su propósito tienden a generar mayor distancia entre la cotidianidad de los ciudadanos y la academia- sino que se visibiliza mediante las posibilidades de proximidad que se gestan para incidir de forma más directa en la comunidad.

Logrando así, que la mirada distante con que la población menos favorecida tiende a percibir la Universidad de los Llanos - Colombia, pueda día a día ser mucho menos utópica y más optimista al tiempo en que el principal ente promotor de pensamiento de un pueblo, fije con más detalle su atención en las necesidades de su región para proyectar la construcción de posibles soluciones conducentes al desarrollo local.

De igual forma esto logra un objetivo de desarrollo humano que despliega de manera progresiva e intencionada, el puente entre las capacidades personales y colectivas de una comunidad, en relación con la interacción personal y dialéctica frente a los ambientes materiales, socioculturales, políticos y económicos que permiten el desarrollo de soluciones problemáticas y facultades que los sujetos enfrentan cotidianamente (Sánchez, 2017). Lo que invita a proyectar acciones que a partir de esfuerzos articulados de pocos se privilegie el bienestar de muchos. 
Por otro lado, cabe mencionar la existencia de una preocupación por el desarrollo entendido en un sentido macro como la inquietud por el estudio de aquello que favorece el progreso y bienestar de todos, sin desconocer que cada comunidad ha tenido su propia fórmula para solventarlo (Aznar \& Barrón, 2017).

Siendo interés de este articulo dar a conocer los aspectos que han incidido en el desarrollo comunitario del barrio la Madrid, desde una mirada basada en la educación física, se considera fundamental tener en cuenta que uno de los obstáculos reflejados es el uso del tiempo libre y la recreación, constituyen en un desafío actual ya que puede transformar las condiciones sociales y culturales de cualquier ambiente (Rodríguez \& González, 2019).

Sin llegar a pensar que la Universidad debe asumir responsabilidades propias del Estado en la lucha contra la pobreza y entendiendo que el desarrollo de un pueblo depende en gran medida de la calidad y compromiso social de su Universidad en términos de transformación de contexto, vale la pena reconocer que según de Souza (2019) "para transformar la realidad de un territorio es fundamental transformar la percepción actual que tienen las comunidades del territorio sobre esa realidad (...)". (p. 93).

En este orden de ideas, la intervención comunitaria realizada por la Universidad de los Llanos en el barrio la Madrid de Villavicencio, a través del proyecto Ocúpate, no había contado con un ejercicio investigativo que le permita develar el nivel de aceptación de la comunidad beneficiaria, así como los logros obtenidos durante su desarrollo, razón por la cual, surgió esta propuesta que determinó un método descriptivo de corte cualitativo para lo que se implementaron como técnicas de recolección de información entrevistas y revisión documental. Para Montalvo et al. "La Responsabilidad Social Universitaria es una estrategia orientada a alcanzar un nivel de excelencia y pertinencia en la calidad del quehacer de las instituciones de Educación Superior en busca de la transformación social para lograr el desarrollo sostenible. (p. 99).

Por otro lado, a manera de conclusión deja ver como el mencionado proyecto de intervención comunitaria viene ganando reconocimiento por parte de la población del barrio la Madrid de Villavicencio (Colombia), lo que ha permitido desvanecer la timidez con que esta población veía a la Universidad, logrando que entre la prospectiva que los habitantes del barrio tienen respecto a sus familias, se encuentre presente la Universidad no solo como fuente directa de potencialización del capital humano por antonomasia, sino como promotora asidua de capital social.

De acuerdo con lo anterior, Castro y Pérez (2017) citado por Carranza, Salazar y Hinojosa (2020) expresan que este tipo de prácticas son resueltas por fases específicas que constituyen una visión amplia fundamentada en la unidad familiar y las relaciones personales, estas estrategias son seguidas por protocolos de acción, planes de intervención y sistemas de mediación con la finalidad de resolver conflictos, interviniendo así mismo profesionales, docentes padres de familia, líderes locales e instituciones.

\section{METODOLOGÍA}

El estudio tuvo un enfoque mixto con un método descriptivo, entendiendo que este tipo de investigaciones permiten el paso de interrogantes de otros proyectos sobre un fenómeno determinado al ofrecer datos sobre su forma y función, además las descripciones catalogan la información observada para que pueda ser utilizada y replicada por otros (Yanez, 2018).

Las técnicas de recolección de información implementadas fueron la revisión documental y 
la entrevista, las cuales se desarrollaron a partir de las siguientes funciones:

-La revisión documental se enfocó en los informes del proyecto ocúpate, el convenio inter-administrativo celebrado entre el Instituto Municipal de Deporte y Recreación - IMDER y la Universidad de los Llanos, al igual que el plan de desarrollo del barrio la Madrid de Villavicencio - Colombia; hay que tener en cuenta que este último es el único documento comunitario construido de forma participativa, el cual contiene lo que la comunidad ha expresado y estipulado a manera de proyección para garantizar el bienestar de su barrio. Iniciativa que los convierte fácilmente en un modelo a seguir para otras comunidades.

-La entrevista estructurada posibilitó el contraste de ideas y apreciaciones frente al proyecto Ocúpate y su intencionalidad de transformación social y contribución al desarrollo comunitario, a su vez dio lugar a expresar apreciaciones que difieren 0 corroboran las dinámicas generadas por el proyecto en mención a partir de la articulación interinstitucional para poder potenciar el capital social del barrio La Madrid. Lo cual se llevó a cabo manteniendo el objetivo de la entrevista, que consistía en recoger información de calidad que permitiese analizar las percepciones diferenciales que han tenido los distintos sujetos muéstrales alrededor del proyecto comunitario Ocúpate.

\section{POBLACIÓN Y MUESTRA}

Se realizó un muestreo por conveniencia, ya que la investigación tuvo la necesidad de especificar a los sujetos que permitieron una mayor recolección de la información, entre ellos se encuentran, el director del IMDER, el presidente de la junta de acción comunal (JAC), una lideresa comunitaria y una habitante de la comunidad, mostrando un total de 4 personas entrevistadas.

\section{PROCEDIMIENTO}

En la práctica de campo se establecieron unos parámetros de recolección de información teniendo en cuenta los instrumentos utilizados en el estudio, para la revisión documental se hizo la respectiva petición a las diferentes entidades que permitieron el acceso a los documentos, dicho de esta manera, la Universidad de los Llanos y el barrio La Madrid dieron aval para analizar los documentos, posteriormente se establecieron lo elementos propios de las categorías de análisis que conllevan a los temas de transformación social, el convenio interadministrativo, el desarrollo del capital humano y social, al igual que la replicación del proyecto a otras comunidades.

Los sujetos participantes concedieron la autorización pertinente para realizar la entrevista estructurada, siendo esta llevada a cabo en sus propios domicilios, exceptuando la del director del IMDER a quien se le entrevisto en su despacho.Resultados 
Tabla 1. Síntesis de las entrevistas

\begin{tabular}{|c|c|c|c|c|}
\hline $\begin{array}{c}\text { Temas de } \\
\text { conversación }\end{array}$ & $\begin{array}{c}\text { Director Instituto } \\
\text { Municipal de Deporte } \\
\text { y Recreación - } \\
\text { IMDER }\end{array}$ & $\begin{array}{l}\text { Líder comunitario } \\
\text { barrio la Madrid }\end{array}$ & $\begin{array}{l}\text { Presidente JAC } \\
\text { barrio la Madrid }\end{array}$ & $\begin{array}{l}\text { Habitante del } \\
\text { barrio la Madrid }\end{array}$ \\
\hline $\begin{array}{l}\text { Transformación } \\
\text { social y } \\
\text { desarrollo } \\
\text { comunitario }\end{array}$ & $\begin{array}{l}\text { "que el programa } \\
\text { Ocúpate tenía } \\
\text { arraigo en la } \\
\text { comunidad del } \\
\text { barrio de la Madrid, } \\
\text { el desarrollo de } \\
\text { las actividades } \\
\text { sobre todo en torno } \\
\text { a los recreativo } \\
\text { había generaron } \\
\text { unos procesos de } \\
\text { cohesión social, que } \\
\text { terminaron siendo } \\
\text { prácticamente ya } \\
\text { un reclamo de la } \\
\text { gente, la gente } \\
\text { quiere que el } \\
\text { programa siga, nos } \\
\text { habían referenciado } \\
\text { la recreo vía } \\
\text { interna, nos habían } \\
\text { referenciado } \\
\text { algunas actividades } \\
\text { recreativas con } \\
\text { jóvenes y querian } \\
\text { que se fortaleciera } \\
\text { esta iniciativa" }\end{array}$ & $\begin{array}{l}\text { “(..) La búsqueda } \\
\text { de los talentos la } \\
\text { cual nos permitió } \\
\text { como identificar esos } \\
\text { niños que tienen } \\
\text { esos potenciales y } \\
\text { igualmente fueron } \\
\text { incentivados de } \\
\text { alguna forma para } \\
\text { que esos sigan estas } \\
\text { actividades que } \\
\text { mejorara la calidad } \\
\text { de vida de todos } \\
\text { aquellos que están } \\
\text { participando, eh } \\
\text { esas capacitaciones } \\
\text { también nos } \\
\text { permitieron el } \\
\text { manejo del tiempo en } \\
\text { cuanto a las nuevas } \\
\text { tecnologías ya que } \\
\text { actual mente a causa } \\
\text { de la pandemia } \\
\text { hemos tenido que } \\
\text { aprender a manejar } \\
\text { estos medios y } \\
\text { estos estudiantes } \\
\text { nos han ayudado } \\
\text { de muchas formas } \\
\text { para que nosotros } \\
\text { aprendamos a } \\
\text { manejar estos medios } \\
\text { tecnológicos que en } \\
\text { muchas ocasiones } \\
\text { no teníamos } \\
\text { conocimiento de cómo } \\
\text { se hacía y ellos muy } \\
\text { amablemente nos } \\
\text { enseñaron (...)” }\end{array}$ & $\begin{array}{l}\text { “a comunidad se } \\
\text { está vinculado } \\
\text { a ellos de } \\
\text { muchas maneras } \\
\text { aportando, } \\
\text { participando y la } \\
\text { verdad son muy } \\
\text { importante, ha } \\
\text { habido un cambio } \\
\text { en la mentalidad } \\
\text { las personas de } \\
\text { que la recreación } \\
\text { en sus vidas es } \\
\text { importante que } \\
\text { la capacitación } \\
\text { en sus vidas es } \\
\text { muy importante, } \\
\text { de la protección } \\
\text { del medio } \\
\text { ambiente es } \\
\text { muy importante, } \\
\text { entonces todas } \\
\text { estas actividades } \\
\text { que hemos } \\
\text { desarrollado. } \\
\text { Ha sido gracias } \\
\text { a la alianza } \\
\text { entre la entre } \\
\text { la Universidad } \\
\text { los llanos con el } \\
\text { proyecto ocúpate } \\
\text { con las Juntas de } \\
\text { Acción Comunal } \\
\text { para el beneficio } \\
\text { de la comunidad” }\end{array}$ & $\begin{array}{l}\text { "yo participé en } \\
\text { los proyectos } \\
\text { ocúpate de la } \\
\text { universidad de } \\
\text { los llanos eh } \\
\text { participe en el } \\
\text { proyecto de } \\
\text { huertas caseras } \\
\text { me pareció una } \\
\text { excelente idea } \\
\text { ese proyecto ya } \\
\text { que nos sirvió a } \\
\text { la comunidad" }\end{array}$ \\
\hline
\end{tabular}




\begin{tabular}{|c|c|c|c|c|}
\hline $\begin{array}{l}\text { articulación } \\
\text { interinstitucional } \\
\text { para } \\
\text { potenciar el } \\
\text { capital social }\end{array}$ & $\begin{array}{l}\text { “hoy la gente } \\
\text { reclama que vuelvan } \\
\text { esos espacios, lo } \\
\text { que quiere decir } \\
\text { que el programa } \\
\text { definitivamente } \\
\text { tocó las fibras } \\
\text { comunitarias, } \\
\text { nosotros lo que } \\
\text { hemos hecho es } \\
\text { firmar un convenio } \\
\text { con la Universidad } \\
\text { de los llanos, un } \\
\text { convenio marco que } \\
\text { nos permite muchas } \\
\text { cosas en materia de } \\
\text { acción comunitaria, } \\
\text { investigación, } \\
\text { pasantías, pero } \\
\text { también un proceso } \\
\text { formativo muy } \\
\text { importante que } \\
\text { se relaciona con } \\
\text { el programa, que } \\
\text { es el diplomado } \\
\text { relacionado con } \\
\text { gestión comunitaria } \\
\text { del deporte y la } \\
\text { recreación (...)” }\end{array}$ & $\begin{array}{l}\text { "Claro que sí, } \\
\text { nuestros habitantes } \\
\text { fueron muy } \\
\text { afortunadas ya que } \\
\text { el proyecto ocúpate } \\
\text { se articuló con la } \\
\text { secretaria de la mujer, } \\
\text { así como con el } \\
\text { IMDER y con muchas } \\
\text { entidades de parte de } \\
\text { la alcaldía las cuales } \\
\text { permitieron potenciar } \\
\text { las capacidades } \\
\text { que tienen nuestras } \\
\text { mujeres del barrio } \\
\text { la Madrid así } \\
\text { como nuestros } \\
\text { niños, jóvenes y } \\
\text { adolescentes" }\end{array}$ & $\begin{array}{l}\text { "Si, inicialmente } \\
\text { todos los } \\
\text { procesos } \\
\text { comunitarios } \\
\text { los hemos } \\
\text { arrancado a nivel } \\
\text { comunitario sí } \\
\text { y a nivel de la } \\
\text { Academia" }\end{array}$ & $\begin{array}{l}\text { "Para ser unidos } \\
\text { para tener un } \\
\text { espacio en qué } \\
\text { invertirnos ya que } \\
\text { manteníamos } \\
\text { demasiados } \\
\text { ocupados en } \\
\text { ese proyecto } \\
\text { se vincularon } \\
\text { los abuelos los } \\
\text { niños hubo más } \\
\text { unión familiar } \\
\text { hubo más unión } \\
\text { con los vecinos } \\
\text { a nosotros en } \\
\text { lo personal nos } \\
\text { sirvió bastante y } \\
\text { quisiéramos que } \\
\text { estos proyectos } \\
\text { siguieran } \\
\text { surgiendo más } \\
\text { a menudo que } \\
\text { implementaran } \\
\text { para nuestra } \\
\text { comunidad lo } \\
\text { del reciclaje que } \\
\text { armaran muchos } \\
\text { proyectos } \\
\text { productivos } \\
\text { para nuestra } \\
\text { comunidad } \\
\text { y nuestros } \\
\text { niños nuestros } \\
\text { ancianos mejor } \\
\text { dicho para toda } \\
\text { la comunidad" }\end{array}$ \\
\hline
\end{tabular}




\begin{tabular}{|c|c|c|c|c|}
\hline $\begin{array}{l}\text { Desarrollo del } \\
\text { capital humano }\end{array}$ & $\begin{array}{l}\text { “(...) vemos que hay } \\
\text { una gran capacidad } \\
\text { de liderazgo de los } \\
\text { líderes comunitarios } \\
\text { del barrio de la } \\
\text { Madrid, que, entre } \\
\text { tantas cosas, yo } \\
\text { puedo compartirles } \\
\text { el documento, nos } \\
\text { han presentado un } \\
\text { plan } \\
\text { relacionado con el } \\
\text { tema deportivo y } \\
\text { su comunidad cosa } \\
\text { que nos parece muy } \\
\text { interesante, que } \\
\text { se hagan planes } \\
\text { comunitarios y } \\
\text { creo que ese es el } \\
\text { aporte del programa } \\
\text { Ocúpate, que } \\
\text { incentivo a la gente a } \\
\text { generar sus propios } \\
\text { planes y proyectos } \\
\text { para su territorio (...)” }\end{array}$ & $\begin{array}{l}\text { "Obvio los habitantes } \\
\text { de la Madrid } \\
\text { aprovecharon al } \\
\text { máximo todas las } \\
\text { capacitaciones que } \\
\text { dieron en cuanto a } \\
\text { las huertas caseras } \\
\text { el taller de screen los } \\
\text { cuales permitieron } \\
\text { que están familias } \\
\text { hoy en día tengan un } \\
\text { nuevo arte tengan } \\
\text { una nueva profesión } \\
\text { tengan una nueva } \\
\text { visibilizacion para } \\
\text { que puedan obtener } \\
\text { unos recursos ya que } \\
\text { por ejemplo en el } \\
\text { taller de screen esas } \\
\text { mujeres aprendieron } \\
\text { a manejar todo lo que } \\
\text { es las técnicas que } \\
\text { permitieron hoy en día } \\
\text { que alguna de ellas } \\
\text { formaran unas micro } \\
\text { empresas y hoy en } \\
\text { día estén participando } \\
\text { con nuestros vecinos } \\
\text { cuando requieran de } \\
\text { estampados y esto ha } \\
\text { permitido que generen } \\
\text { un ingreso entonces } \\
\text { pues la verdad } \\
\text { ocúpate ha venido } \\
\text { a mejorar la calidad } \\
\text { de vida de todos } \\
\text { nosotros" }\end{array}$ & $\begin{array}{l}\text { "(...) el programa } \\
\text { ocúpate varios } \\
\text { proyecto, } \\
\text { también el de } \\
\text { huertas caseras, } \\
\text { entonces esto ha } \\
\text { servido mucho de } \\
\text { que las personas } \\
\text { que participan } \\
\text { se fortalezcan } \\
\text { académicamente, } \\
\text { por ejemplo, en el } \\
\text { de huertos casera } \\
\text { hay } 82 \text { familias } \\
\text { que se vieron } \\
\text { beneficiadas son } \\
82 \text { familias, que } \\
\text { ya saben hacer } \\
\text { fuerzas en sus } \\
\text { propias viviendas } \\
\text { antes no lo } \\
\text { sabían hacer y } \\
\text { si lo hicieron y } \\
\text { lo aprendieron } \\
\text { en el tiempo y } \\
\text { pandemia, eso } \\
\text { es importante } \\
\text { que a través de } \\
\text { la virtualidad } \\
\text { pudimos dar de } \\
\text { alguna u otra } \\
\text { manera y esa } \\
\text { capacitación para } \\
\text { ellos tuvieran ese } \\
\text { conocimiento } \\
\text { y pudieran } \\
\text { desarrollarlo en } \\
\text { sus vivienda (...)” }\end{array}$ & $\begin{array}{l}\text { "Definitivamente } \\
\text { el proyecto } \\
\text { ocúpate de la } \\
\text { universidad } \\
\text { Unillanos ha } \\
\text { permitido una } \\
\text { mejor calidad } \\
\text { de vida para } \\
\text { la comunidad } \\
\text { de la Madrid } \\
\text { y sería muy } \\
\text { importante que } \\
\text { este proyecto se } \\
\text { llevara a cabo en } \\
\text { todos los barrios } \\
\text { de Villavicencio" }\end{array}$ \\
\hline
\end{tabular}




\begin{tabular}{|c|c|c|c|c|}
\hline $\begin{array}{l}\text { Replicación } \\
\text { del proyecto } \\
\text { en otras } \\
\text { comunidades }\end{array}$ & 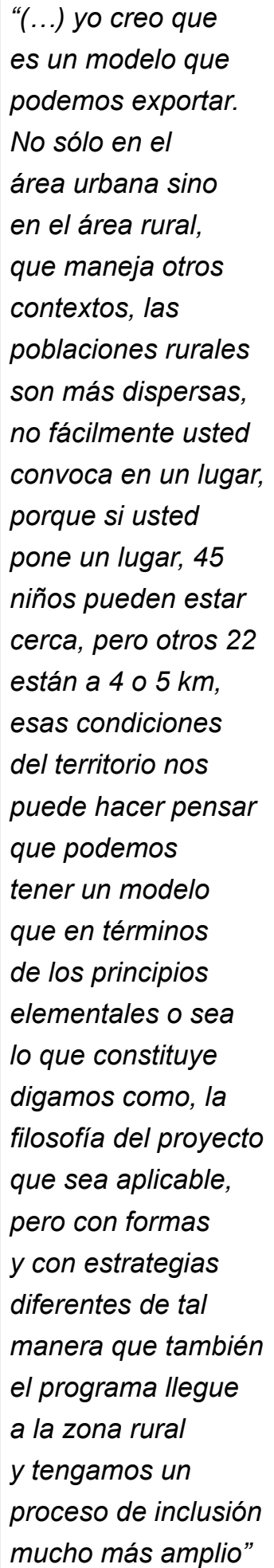 & $\begin{array}{l}\text { “También me gustaría } \\
\text { que se unieran } \\
\text { más instituciones } \\
\text { como la alcaldía, } \\
\text { la gobernación, } \\
\text { secretarias, para } \\
\text { que vincularan y } \\
\text { nos hicieran muchos } \\
\text { más proyectos } \\
\text { productivos para } \\
\text { nuestra comunidad y } \\
\text { sociedad con el fin de } \\
\text { que la Madrid tenga } \\
\text { un sector más visto } \\
\text { ante la comunidad ya } \\
\text { que debido a tanta } \\
\text { problemática con las } \\
\text { drogas con los niños } \\
\text { que no están en el } \\
\text { colegio porque acá } \\
\text { han estado bastante } \\
\text { difícil los cupos } \\
\text { entonces hay mucho } \\
\text { niños desocupado } \\
\text { y la idea es que se } \\
\text { unan todas estas } \\
\text { instituciones a sacar } \\
\text { adelante” }\end{array}$ & $\begin{array}{l}\text { Sí, claro, } \\
\text { indiscutiblemente } \\
\text { porque es } \\
\text { que todos los } \\
\text { proyectos deben } \\
\text { retener su } \\
\text { componente } \\
\text { académico. Eso } \\
\text { es importante } \\
\text { para que hay } \\
\text { una socialización } \\
\text { halla una } \\
\text { ilustración, halla } \\
\text { una inducción } \\
\text { de cómo se } \\
\text { deben realizar } \\
\text { los procesos y } \\
\text { que como se } \\
\text { puede vincular } \\
\text { la comunidad } \\
\text { y como se } \\
\text { ve beneficia, } \\
\text { entonces pienso } \\
\text { que el tema } \\
\text { académico en } \\
\text { todo proyecto } \\
\text { es importante y } \\
\text { por eso debería } \\
\text { replicarse este } \\
\text { modelo en } \\
\text { muchas otras } \\
\text { ciudades. }\end{array}$ & $\begin{array}{l}\text { "Nosotros } \\
\text { logramos } \\
\text { mejorar nuestra } \\
\text { calidad de vida } \\
\text { muchos barrios } \\
\text { también pueden } \\
\text { imitar y llegar a } \\
\text { desarrollar sus } \\
\text { habilidades que } \\
\text { tienen por medio } \\
\text { de todas estas } \\
\text { actividades que } \\
\text { este proyecto } \\
\text { nos ha traído } \\
\text { en el barrio de } \\
\text { la Madrid esto } \\
\text { ha permitido } \\
\text { también que } \\
\text { nosotros } \\
\text { conozcamos } \\
\text { de cerca las } \\
\text { problemáticas } \\
\text { la Madrienen } \\
\text { nuestra } \\
\text { comunidad y } \\
\text { podamos de } \\
\text { una u otra forma } \\
\text { ayudarles para } \\
\text { que mejore } \\
\text { tanto como la } \\
\text { convivencia } \\
\text { demo la calidad de todas } \\
\text { lasonas que } \\
\text { latan barrio }\end{array}$ \\
\hline
\end{tabular}

Fuente: Elaboración propia 
Matriz de revisión documental

\begin{tabular}{|c|c|c|c|}
\hline $\begin{array}{l}\text { DOCUMENTOS } \\
\text { CATEGORIAS } \\
\text { DEANAISIS }\end{array}$ & INFORMES & $\begin{array}{c}\text { PLAN DE } \\
\text { DESARROLLO DE } \\
\text { LAMADRID }\end{array}$ & $\begin{array}{l}\text { CONVENIO INTER- } \\
\text { INSTITUCIONAL }\end{array}$ \\
\hline $\begin{array}{l}\text { TIEMPO } \\
\text { LIBRE }\end{array}$ & $\begin{array}{l}\text { Frente al uso del tiempo libre es im- } \\
\text { portante mencionar que el proyecto } \\
\text { Ocúpate generó diferentes estrate- } \\
\text { gias ofreciendo a la comunidad la } \\
\text { posibilidad de participar constante- } \\
\text { mente en distintas actividades } \\
\text { orientadas en ambientes lúdicos y } \\
\text { con propósitos pedagógicos en las } \\
\text { que a lo largo del año } 2019 \text { más de } \\
\text { setecientas personas se beneficiar- } \\
\text { on permanentemente. } \\
\text { Con relación al año } 2020 \text { es de } \\
\text { resaltar la forma en que se modi- } \\
\text { ficaron las estrategias para incor- } \\
\text { porarse en el ambiente de la virtu- } \\
\text { alidad (por motivos de aislamiento } \\
\text { social derivado de la pandemia por } \\
\text { covid19) } \\
\text { Y dentro de los indicadores de } \\
\text { asistencias a las reuniones de } \\
\text { planeación participativa para la } \\
\text { construcción de estrategias de } \\
\text { intervención -lo cual también se } \\
\text { desarrolló en el tiempo libre de los } \\
\text { participantes- fue relevante el com- } \\
\text { promiso de la mayoría de presi- } \\
\text { dentes de JAC del barrio y líderes } \\
\text { comunitarios, así como la incorpo- } \\
\text { ración del Instituto Municipal de De- } \\
\text { porte y Recreación. }\end{array}$ & $\begin{array}{l}\text { El barrio La Madrid es la única } \\
\text { comunidad a nivel municipal } \\
\text { que cuenta con plan de desar- } \\
\text { rollo, por tal motivo al realizar } \\
\text { el análisis documental se en- } \\
\text { contró que dentro del parágra- } \\
\text { fo } 4 \text { se relaciona el proyecto } \\
\text { recreo vía de La Madrid como } \\
\text { un factor para el aprovechami- } \\
\text { ento del tiempo libre de la po- } \\
\text { blación del barrio que estudia } \\
\text { o trabaja, del mismo modo } \\
\text { Se menciona al proyecto } \\
\text { Ocúpate como apoyo para } \\
\text { brindarle a la comunidad un } \\
\text { espacio en el que se realicen } \\
\text { actividades físicas, disciplinas } \\
\text { deportivas como el fútbol volei- } \\
\text { bol patinaje, zumba y demás } \\
\text { actividades que contemplen el } \\
\text { esparcimiento y la formación } \\
\text { integral de la comunidad. }\end{array}$ & $\begin{array}{l}\text { La Celebración de un convenio } \\
\text { interadministrativo entre la Univer- } \\
\text { sidad de los Llanos y el IMDER, } \\
\text { motivado entre otras cosas por } \\
\text { los resultados del proyecto OCÚ- } \\
\text { PATE estableció como objeto: } \\
\text { "Aunar esfuerzos entre el Insti- } \\
\text { tuto Municipal de Deporte y Rec- } \\
\text { reación (IMDER) y la Universidad } \\
\text { de los Llanos (Unillanos) para for- } \\
\text { mar agentes comunitarios depor- } \\
\text { tivos y potenciar las escuelas de } \\
\text { iniciación deportiva en el municipio } \\
\text { de Villavicencio" en el cual se dejó } \\
\text { estipulado la necesidad de contra- } \\
\text { tar dos "Monitores (estudiantes de } \\
\text { decimo semestre de licenciatura } \\
\text { en educación física) en deporte } \\
\text { comunitario encargados de hacer } \\
\text { apertura del proyecto OcÚPATE } \\
\text { en dos barrios vulnerables de Vil- } \\
\text { lavicencio durante dos meses. } \\
\text { Lo anterior como una apuesta a } \\
\text { la creación de alternativas de ocu- } \\
\text { pación del tiempo libre en otros } \\
\text { escenarios del municipio de Vil- } \\
\text { lavicencio. }\end{array}$ \\
\hline $\begin{array}{l}\text { CAPITAL } \\
\text { SOCIAL }\end{array}$ & $\begin{array}{l}\text { Algunos apartes encontrados en } \\
\text { los informes del proyecto Ocúpate } \\
\text { dejan ver como en el año } 2019 \\
\text { se otorgan a este algunos recono- } \\
\text { cimientos por parte de la prensa, de } \\
\text { igual forma otros medios de comu- } \\
\text { nicación a nivel institucional como } \\
\text { "comunicaciones Unillanos" dedi- } \\
\text { caron notas periodísticas mostran- } \\
\text { do la labor del proyecto Ocúpate, }\end{array}$ & $\begin{array}{l}\text { Es necesario mencionar que } \\
\text { siendo los presidentes de } \\
\text { juntas de acción comunal del } \\
\text { barrio la Madrid de Villavi- } \\
\text { cencio con el acompañami- } \\
\text { ento de líderes comunitarios, } \\
\text { quienes tuvieron la iniciativa } \\
\text { de co-crear el plan de de- } \\
\text { sarrollo de su propio barrio } \\
\text { y que por decisión unánime }\end{array}$ & $\begin{array}{l}\text { Las alianzas interinstitucionales } \\
\text { materializadas en un convenio } \\
\text { interadministrativo que posibilita la } \\
\text { conformación de un grupo de ge- } \\
\text { stores recreo-deportivos del mu- } \\
\text { nicipio de Villavicencio, sumadas } \\
\text { a los esfuerzos de los lideres co- } \\
\text { munitarios y presidentes de JAC, } \\
\text { secretaría de la mujer, así como al } \\
\text { comercio del barrio la Madrid de }\end{array}$ \\
\hline
\end{tabular}




\begin{tabular}{|c|c|c|c|}
\hline & $\begin{array}{l}\text { a su vez el periódico Llano } 7 \text { Días } \\
\text { destacó que las estrategias emplea- } \\
\text { das en el marco del proyecto fueron } \\
\text { adecuadas para la ocupación del } \\
\text { tiempo libre extraescolar de los } \\
\text { habitantes del barrio La Madrid de } \\
\text { Villavicencio, apoyándose en el ob- } \\
\text { jetivo de impacto que genera este } \\
\text { tipo de estrategias para el contexto } \\
\text { social. } \\
\text { La articulación con el programa de } \\
\text { licenciatura en producción agropec- } \\
\text { uaria, con el IMDER, con las JAC, } \\
\text { con el comercio del sector, líderes } \\
\text { comunitarios, con secretaría de la } \\
\text { mujer, con líderes de la comunidad } \\
\text { mediante el apoyo brindado para } \\
\text { fortalecer proyectos de carácter } \\
\text { endógenos }\end{array}$ & $\begin{array}{l}\text { hayan decidido incorporar el } \\
\text { proyecto Ocúpate da mues- } \\
\text { tra del fortalecimiento paula- } \\
\text { tino del capital social, el cual } \\
\text { se fortalece aún más con } \\
\text { la vinculación de diferentes } \\
\text { dependencias de la alcaldía } \\
\text { municipal }\end{array}$ & $\begin{array}{l}\text { Villavicencio, se vienen convirtien- } \\
\text { do en un engranaje reconstructor } \\
\text { de tejido social. } \\
\text { Que al actuar de manera sinérgica } \\
\text { facilita el alcance de los objetivos } \\
\text { comunitarios gestando el capital } \\
\text { social. }\end{array}$ \\
\hline $\begin{array}{l}\text { CAPITAL } \\
\text { HUMANO }\end{array}$ & $\begin{array}{l}\text { Los informes sistemáticos del } \\
\text { proyecto Ocúpate evidencian que } \\
\text { los niños participantes de las es- } \\
\text { trategias implementadas, desarrol- } \\
\text { laron diferentes habilidades sociales } \\
\text { y por otro lado, } 83 \text { adultos demos- } \\
\text { traron haber adquirido conocimien- } \\
\text { tos relacionados con elaboración } \\
\text { de huertas caseras, por lo que la } \\
\text { Universidad de los llanos les cer- } \\
\text { tificó, de igual forma } 38 \text { adultos } \\
\text { participaron en un proceso de ca- } \\
\text { pacitación en técnicas de screem } \\
\text { (estampación de camisetas) como } \\
\text { alternativa de ocupación produc- } \\
\text { tiva del tiempo libre y un grupo de } \\
\text { quince personas entre adolescentes } \\
\text { jóvenes y adultos se capacitaron } \\
\text { en liderazgo recreativo lo que ha } \\
\text { posibilitado garantizar la capacidad } \\
\text { instalada, también se les extendió } \\
\text { la opción de recibir una conferen- }\end{array}$ & $\begin{array}{l}\text { La creación del centro de in- } \\
\text { tegración cultural del barrio la } \\
\text { Madrid, en el cual el proyecto } \\
\text { Ocúpate tuvo una partici- } \\
\text { pación directa especialmente } \\
\text { con la creación participativa } \\
\text { de su documento soporte, } \\
\text { se constituye como uno de } \\
\text { los logros más destacados } \\
\text { por el proyecto y uno de los } \\
\text { propósitos más ambiciosos } \\
\text { del plan de desarrollo del bar- } \\
\text { rio en mención. } \\
\text { Pues de acuerdo con lo es- } \\
\text { tipulado frente al centro de } \\
\text { integración cultural, este se } \\
\text { concibe como un escenario } \\
\text { en el que se encontrara } \\
\text { variedad de alternativas de } \\
\text { ocupación del tiempo libre } \\
\text { enmarcadas en los ámbitos } \\
\text { cultural, deportivo, recreativo, }\end{array}$ & $\begin{array}{l}\text { Teniendo presente que el objeto } \\
\text { del convenio pretende } \\
\text { "Aunar esfuerzos entre el Insti- } \\
\text { tuto Municipal de Deporte y Rec- } \\
\text { reación (IMDER) y la Universidad } \\
\text { de los Llanos (Unillanos) para } \\
\text { formar agentes comunitarios de- } \\
\text { portivos y potenciar las escuelas } \\
\text { de iniciación deportiva en el mu- } \\
\text { nicipio de Villavicencio" es claro } \\
\text { que el proceso de formación de } \\
\text { gestores so constituye como uno } \\
\text { de los elementos más importantes } \\
\text { de esta alianza interinstitucional } \\
\text { privilegiando el fortalecimiento del } \\
\text { capital humano. En el mismo sen- } \\
\text { tido, al ofrecer a dos estudiantes la } \\
\text { oportunidad de replicar el proyecto } \\
\text { Ocúpate en otros escenarios del } \\
\text { municipio se amplía la opción de } \\
\text { continuar la dinámica de poten- }\end{array}$ \\
\hline
\end{tabular}


cia sobre emprendimiento, para lograr mucho de lo anterior fue necesario iniciar capacitándose en manejo básico de plataformas digitales como zoom y Meet, ya que a través de encuentro virtuales se desarrollaron varias de estas capacitaciones.

Frente a las encuestas de satisfacción del proyecto de intervención, se encontró que el $100 \%$ de las personas participantes fueron externas, de igual manera el $54 \%$ de las personas encuestadas mencionaron que fue bueno el proyecto social y el $46 \%$ les pareció excelente correlacionándose con la calificación de las actividades dentro del proyecto el $54 \%$ les pareció excelente y el $46 \%$ bueno artístico entre otros, convirtiéndose en un verdadero centro potenciador de capital humano para los habitantes del cada una de los etapas del barrio. ciación del capital humano en torno a la ocupación productiva del tiempo libre.

\section{DISCUSIÓN}

El proceso de re contextualización a partir de la ocupación productiva del tiempo libre enmarcada en el proyecto Ocúpate, son el resultado de un ejercicio sinérgico entre los habitantes del barrio la Madrid y la Universidad de los Llanos, teniendo su interés en esta comunidad; al contrastarlo con otros estudios como el de Veracierto (2020) realizado en el barrio Los Naranjos determinó que la participación ciudadana es positiva frente a las actividades de asfaltado en las calles y arreglo en el sistema de aguas servidas entre otros, motivando el progreso social y el fortaleciendo el desarrollo humano dentro de la misma comunidad. Todos estos proyectos tienen como finalidad aprovechar las potencialidades creadoras de los miembros de la comunidad potenciando cambios a favor de sus intereses y necesidades (Armenteros \& Padrón, 2018).

La importancia de repensar las construcciones ontoepistemologicas que son aprovechadas en la ejecución de proyectos comunitarios, se expresa en los referentes normativos que hay dentro de la misma comunidad por lo tanto las áreas del saber y el compromiso de los actores sociales se reflejan en las actitudes que se muestran al momento de participar en las actividades asumiendo aprendizajes pedagógicos para apoyar en la transformación del contexto que involucra a los actores socioeducativas como la universidad y la sociedad en general (Guevara, 2017).

Las experiencias accionarias aplicadas permite compartir las vivencias y realidades que se asemejan a las diferentes comunidades lo cual genera una necesidad de participación activa de los diferentes actores en los proyectos comunitarios, teniendo como resultado experiencias gratificantes y satisfactorias en términos profesionales y personales que es acompañada por emociones y sentimientos que se despiertan en los diferentes colectivos territoriales, esta expresión es evoca a los proyectos comunitarios de Romero y Zabala (2019) apoyando en la hipótesis qué los 
proyectos comunitarios no sólo cambian las realidades de los pobladores sino que también la realidad de los organizadores.

El estudio de Góngora (2020) establece que el trabajo comunitario es un modo eficaz para avanzar en el progreso de las comunidades que siempreestá en búsqueda de un diseño apropiado del proyecto de transformación local los autores que se encuentran participando puntualizan que los grupos de trabajo tengan compromisos importantes para lograr la participación de los ciudadanos. Esta participación se puede convertir una red de trabajo intercomunitario en la que se vinculan inclusive comunidades vecinas y de cooperación interinstitucional que le otorga un valor social importante en la consecución y cumplimiento de objetivos locales ahora bien el papel que juega las instituciones participantes se visibiliza en la confianza que adquiere para el desarrollo de las comunidades constituyéndose en un elemento de capital social en el ámbito comunitario (Ayaviri, Quispe \& Borja, 2017).

De acuerdo a lo anterior, Mena (2017) difiere en la posibilidad de que haya cumplimiento de los objetivos comunitarios si los participantes tienen como objetivo recoger dinero para las celebraciones, puesto que pocos se vinculan a las actividades que les permitan discutir y proponer solucionar los problemas comunitarios, enfocándose en los objetivos monetarios y no de desarrollo local.

Por otro lado, los proyectos de carácter comunitario y de participación activa de los integrantes hace que el desarrollo sostenible sea efectivamente articulada constructivamente y de forma sinérgica por los ejes sociales económicos e inclusive ecológicos, teniendo en cuenta que el eje económico debe estar basado exclusivamente en el conocimiento de igual manera el eje social incurre en la socio-formación es decir la vinculación de la comunidad las organizaciones y la participación educativa, con esta articulación el desarrollo social sostenible se puede construir de manera equilibrada para lograr metas propuestas en común (Hernández, Tobón, Salas \& Carno, 2019)

Por último, Quintero (2017) argumenta que la participación de los proyectos comunitarios se hace de manera activa, realizando presentaciones artísticas, ayudas logísticas y de gestión, demostrando que los ciudadanos pueden generar experiencias a partir de iniciativas como movilizaciones masivas de carácter participativo, siendo de vital importancia para constituir el capital social y el desarrollo humano dentro de las comunidades. Estas propuestas mejoran la convivencia que se sitúan la mayoría de los territorios que fortalecen, la organización local y articulan los espacios de relación, participación y el trabajo intersectorial (Gutiérrez, Romero, \& Marchioni, 2020).

\section{CONCLUSIONES}

La constancia, el carácter participativo y el compromiso de los diferentes actores son los ingredientes fundamentales para garantizar que un proceso de intervención comunitaria se desarrolle y madure lo suficiente, logrando que los resultados esperados puedan evidenciarse mediante las dinámicas apropiadas por la comunidad beneficiaria reconocidas como capacidad instalada, lo que simultáneamente permitirá que se resignifiquen como seres sociales capaces de transformar su propia realidad.

Es evidente que el proceso de intervención social adelantado por la Universidad de los Llanos en el barrio la Madrid de Villavicencio, ha sido crucial en la formulación colectiva de estrategias de ocupación lúdica y productiva del tiempo libre de sus habitantes, sin desconocer que las principales ideas para tal fin, han surgido de manera endógena como resultado de una serie de necesidades que la comunidad venia expresando años atrás y que la implementación del proyecto ocúpate funcionó como dinamizador 
y articulador de los diferentes actores que materializaron dichas estrategias.

El proceso de intervención comunitaria adelantado por el proyecto Ocúpate de la Universidad de los Llanos en el barrio la Madrid de Villavicencio - Colombia ha generado resultados contribuyentes en su desarrollo comunitario, lo cual se ha logrado a partir de la implementación de estrategias construidas de manera participativa con los diferentes actores de la comunidad, siendo esto último uno de los elementos más significativos de dicho proceso.

La potenciación del capital humano de los habitantes del barrio la Madrid de Villavicencio - Colombia, fue evidente en una pequeña parte de la población, la cual a partir de los procesos de capacitación enmarcados en las estrategias de manejo de plataformas digitales, elaboración de huertas caseras, taller de técnicas de screem (estampación), como alternativa de ocupación productiva del tiempo libre, apropiaron conocimientos elementales que asumieron como herramientas para proyectar nuevas alternativas frente a la ampliación de sus posibilidades de ingreso económicos.

El alcance de los objetivos del proyecto de intervención Ocúpate, relacionados al fortalecimiento del capital social del barrio la Madrid, se dio en buena medida gracias su carácter recurrente, lo cual, se constituye como uno de los aspectos clave del proceso reflejado en la capacidad instalada que se visibiliza mediante la articulación del IMDER, Universidad de los Llanos, Juntas de acción comunal del barrio la Madrid, Lideres comunitario, secretará de medio ambiente, ONG, sector comercio del barrio, etc.

\section{REFERENCIAS BIBLIOGRÁFICAS}

Armenteros Rojas, A., \& Padrón Iglesias, Á. P. (2018). Los proyectos comunitarios y su influencia en la calidad de vida de las personas mayores. Revista de Ciencias Médicas de Pinar del Río, 22(2), 185195.

Ayaviri, V. D., Quispe, G. M., \& Borja, M. E. (2017). El Capital Social en el desarrollo local comunitario. Un estudio en comunidades rurales de Bolivia. Revista Espacios, 38(43).

Aznar Minguet, P., \& Barrón Ruiz, Á. (2017). El desarrollo humano sostenible: un compromiso educativo.

Carranza, K., Salazar, M., \& Hinojosa, M. (2020). Trabajo social para la participación comunitaria en una institución educativa de Colombia. Orbis: revista de Ciencias Humanas, 16(47), 66-76.

de Souza Silva, J. (2019). El Buen Vivir rural y la construcción colectiva de Proyectos de Vida Comunitarios. Cuban Journal of Agricultural Science, 53(1), 91-101.

de Veracierto, L. M. C. (2020). Proyectos comunitarios: Una experiencia didáctica en Formación Comunitaria. Revista Scientific, 5(15), 209-228.

Góngora, C. D. L. Á. G. (2020). Trabajo comunitario: eje esencial en la gestión cultural comunitaria. Didasc@ lia: Didáctica y Educación ISSN 22242643, 11(1), 190-200.

Guevara, C. (2017). Servicio comunitario, aprendizaje-servicio y formación social: claves para la construcción de espacios transformativos universitarios. REDHECS, 23(2), 80105. 
Gutiérrez, M. R., Romero, C. G., \& Marchioni, M. (2020). Una década trabajando por la mejora de la convivencia: El Proyecto de Intervención Comunitaria Intercultural. Revista Madrileña de Salud Pública, 3(9), 1-7.

Hernández, L. G. J., Tobón, S., SalasRazo, G., \& Carno, A. E. J. (2019). Desarrollo sostenible: educación y sociedad. $M+A$, revista electrónica de medioambiente, 20(1), 54-72.

Mena, N. P. (2017). Los estudiantes de secundaria y el vínculo comunitario. Un estudio en tres instituciones educativas de secundaria en Colombia. Educación y Humanismo, 19(32), 67-87.

Montalvo Morales, J. A., Villanueva Armenteros, Y., Armenteros Acosta, M. D. C., Gómez Gutiérrez, E. L., \& Cervantes Ávila, Y. G. (2017). Participación Comunitaria $Y$ Responsabilidad Social Universitaria: Estudio De Caso (Community Participation and Social Sphere of University Social Responsibility). Revista Global de Negocios, 5(5), 99-111.

Quintero, J. S. (2017). La animación sociocultural como enfoque de intervención comunitaria con el adulto mayor. Poiésis, 1(33), 15-20.

Rodríguez, B. M., \& González, J. B. (2019). Actividades deportivas-recreativas comunitarias para mejorar el estilo de vida en jóvenes. Lecturas: Educación Física y Deportes, 23(248), 149-163.

Romero, D. M., \& Zavala, J. J. A. (2019). Proyectos Comunitarios en el proceso de Transformación Social de la Universidad. CIENCIAMATRIA, 5(9), 151-167.
Sánchez-Vidal, A. (2017). Empoderamiento, liberación y desarrollo humano. Psychosocial Intervention, 26(3), 155-163.

Yanez, D. (2018). Método descriptivo: características, etapas y ejemplos. Obtenido de https://www. lifeder. com/metodo-descriptivo. 